\title{
Cognitive reserve mediates the severity of certain neuropsychological deficits related to cocaine use disorder.
}

Sampedro-Piquero, P.; Vicario, S.; Pérez-Rivas, A.; Ladrón de Guevara-Miranda, D.; Santín, L.

Introduction: The concept of cognitive reserve $(\mathrm{CR})$ is being considered in the field of substance use disorder (SUD) by observing that there are individuals whose brain alterations are not related to the cognitive symptomatology they present. Aims: Our aims were to characterise the possible neuropsychological deficits in a sample of subjects with SUD compared to a control group and to determine whether the degree of $\mathrm{CR}$ is a mediator in the cognitive functioning of these patients. Methods: To perform these objectives, the study involved a sample of subjects with SUD in outpatient treatment and a control group. A CR questionnaire and a comprehensive neuropsychological assessment were administered, and we also collected data related to drug consumption and psychological well-being. Results: The SUD group showed poorer performance compared to the control group in several cognitive domains (attention, declarative memory, executive functions and emotional perception), as well as in psychological comfort. Interestingly, we observed that the deficits found in attention, declarative memory and executive functions were mediated by the CR level of the participants, an effect that we did not observe in the rest of the variables registered. Conclusion: Our results suggest that long-term drug consumption leads to cognitive deficits and affects the psychological well-being of the subjects. Moreover, the CR should be taken into account during the assessment and rehabilitation of patients with SUD due to its protective role against certain neuropsychological deficits. 